\title{
Two High-Nutrient Low-Chlorophyll phytoplankton assemblages: the tropical central Pacific and the offshore Perú-Chile Current
}

\author{
F. Gómez ${ }^{1}$, H. Claustre ${ }^{2,3}$, P. Raimbault ${ }^{1}$, and S. Souissi ${ }^{4}$ \\ ${ }^{1}$ Laboratoire d'Océanographie et de Biogéochimie, CNRS UMR 6535, Centre d'Océanologie de Marseille, Université de la \\ Méditerranée, 163 avenue de Luminy, 13288 Marseille, France \\ ${ }^{2}$ UPMC Univ. Paris 06, UMR 7093, Laboratoire d'Océanographie de Villefranche, 06230 Villefranche-sur-Mer, France \\ ${ }^{3}$ CNRS, UMR 7093, LOV, 06230 Villefranche-sur-Mer, France \\ ${ }^{4}$ Station Marine de Wimereux, Université des Sciences et Technologies de Lille-Lille1, FRE 2816 ELICO CNRS, 28 avenue \\ Foch, BP 80, 62930 Wimereux, France
}

Received: 11 April 2007 - Published in Biogeosciences Discuss.: 14 May 2007

Revised: 5 October 2007 - Accepted: 27 November 2007 - Published: 14 December 2007

\begin{abstract}
The phytoplankton ( $>15 \mu \mathrm{m})$ composition and abundance was investigated along a $\sim 8000 \mathrm{~km}$ transect between the Marquesas Islands Archipelago and the Chilean coasts off Concepción. In the southern limit of the central Equatorial Pacific (at $8^{\circ} \mathrm{S}, 141^{\circ} \mathrm{W}$ ), in High-Nutrient LowChlorophyll (HNLC) warm waters, the microphytoplankton assemblage was dominated by the lightly silicified diatoms Pseudo-nitzschia delicatissima and Rhizosolenia bergonii. The morphology of these species, a small pennate diatom that exhibited a tendency to form "ball of needles" clusters and large centric diatom ( $>500 \mu \mathrm{m}$ long), are interpreted as two anti-grazing strategies in an environment dominated by small micrograzers. Surprisingly, this a priori typical HNLC phytoplankton assemblage was also found in the temperate offshore waters of the Perú-Chile Current between 2000 and $600 \mathrm{~km}$ off Chile. This observation suggests that a common set of environmental factors (obviously other than temperature and salinity) are responsible for the establishment and maintaining of this distinctive phytoplankton in these geographically and hydrologically distant regions. Both regions are characterized by a surface nitrate-silicic acid ratio ranging from 1-3. Occasionally Rhizosolenia bergonii showed frustules anomalously fragmented, likely the result of extreme weakly silicified phytoplankton. We suggest that silicon deficiency may be responsible of the occurrence of HNLC phytoplankton assemblage in the tropical central Pacific as well as the offshore Perú-Chile Current during the austral summer.
\end{abstract}

Correspondence to: F. Gómez

(fernando.gomez@fitoplancton.com)

\section{Introduction}

The equatorial upwelling region in the central and eastern Pacific constitutes a large area of the so-called the High Nutrient Low Chlorophyll (HNLC) waters, representing a strong source of $\mathrm{CO}_{2}$ to the atmosphere (Chavez and Toggweiler, 1995). Grazing control, ammonium inhibition of nitrogen uptake, limitations in silicic acid, micronutrient (iron) or light, lack of neritic bloom-forming diatoms among other hypothesis have been invoked to explain these paradoxical HNLC conditions (Chavez et al., 1991; Coale et al., 1996; Dugdale and Wilkerson, 1998). The microscope observations in the upwelling region of the central and eastern equatorial Pacific revealed a recurrent phytoplankton assemblage mainly composed of the small pennate diatom Pseudo-nitzschia delicatissima and the large centric diatom Rhizosolenia bergonii (Hasle, 1960).

The iron-hypothesis has challenged new studies in the equatorial upwelling region that have been focused, in particular, on biogeochemical fluxes and parameters such as biomass estimates and rate measures of primary and secondary production following the iron fertilization. These studies were nevertheless accompanied by scarce investigations on the large phytoplankton composition (Kaczmarska and Fryxell, 1994; Iriarte and Fryxell, 1995). They were often limited to identifications at suprageneric levels (Chavez and Buck, 1990) or focused on the species identification during iron-enriched incubation experiments (Fryxell and Kaczmarska, 1995).

The Perú-Chile or Humboldt Current originates when a part of the water that flows toward the east across the subAntarctic Ocean is deflected toward the north as it approaches South America. It flows northward along the coast

Published by Copernicus Publications on behalf of the European Geosciences Union. 


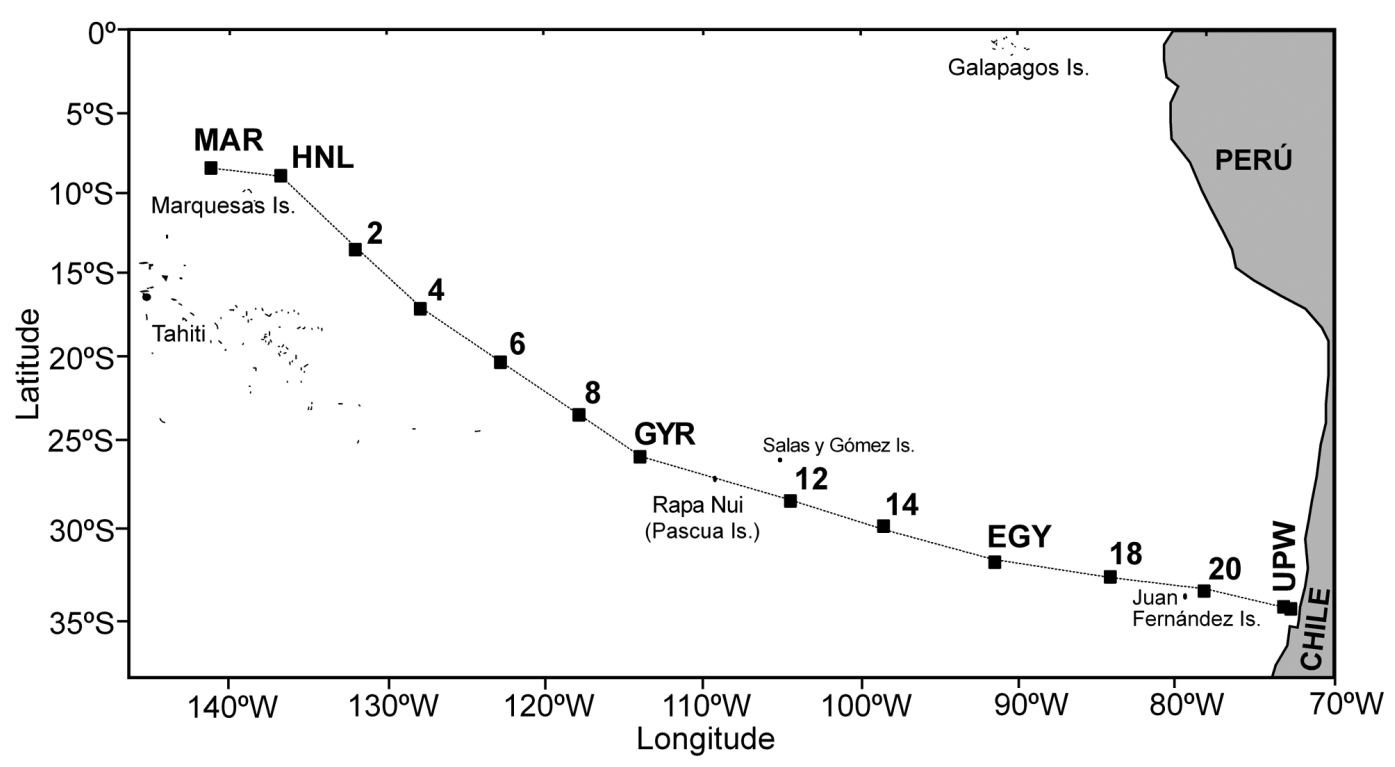

Fig. 1. Map of the stations occupied (solid squares) in the SE Pacific Ocean.

of Chile, Perú and Ecuador, and is associated with the economically most important coastal upwelling of the world's oceans (Longhurst, 1998). The coastal phytoplankton of the region is well documented (e.g. Rivera, 1983; Avaria and Muñoz, 1987). In contrast, the phytoplankton from the open waters of SE Pacific is one of the least known and documented of the world's oceans (Balech, 1962), reflecting the general undersampling of this remote area (Longhurst, 1998; Claustre and Maritorena, 2003). Very few recent investigations have been dedicated to the study of the picophytoplankton in the subtropical SE Pacific in latitudinal transects crossing the equator (DiTullio et al., 2003) or a few surface net samples with scarce detail on the phytoplankton specific composition (Hardy et al., 1996).

Within this context, we examined the composition and abundance of phytoplankton in relation to physical and chemical properties in the SE Pacific Ocean as part of a multidisciplinary survey along a transect of $\sim 8000 \mathrm{~km}$ covering contrasted trophic conditions: HNLC waters of the Marquesas Islands Archipelago, hyper oligotrophic waters of the South Pacific Gyre (SPG), and more eutrophic conditions in the Perú-Chile Current (PCC) and the Chilean coastal upwelling. The present study focuses on the HNLC phytoplankton assemblage (HNLC-PA) near the Marquesas Islands Archipelago, the southern limit of the equatorial upwelling region that unexpectedly was also observed in a vast region of the temperate waters of the SE Pacific off Chile. The similarity in HNLC phytoplankton assemblage between two distant geographical and hydrological regions is analyzed and interpreted in the context of possible driving factors precluding this a priori paradoxical situation.

\section{Methods}

The data were collected during the BIOSOPE (Biogeochemistry and Optics South Pacific Experiment) cruise on board R/V L'Atalante from 26 October-12 December 2004. The ship was equipped with a $75 \mathrm{kHz}$ RDI Acoustic Doppler Current Profiler (ADCP). Water sampling and measurements of temperature and salinity were made using a SeaBird SBE 911plus CTD/Carousel system fitted with an in situ Aquatracka III Chelsea fluorometer and Niskin bottles. The percentage of surface irradiance at each depth was calculated from the underwater PAR (Photosynthetic Active Radiation, $400-700 \mathrm{~nm}$ ) profiles performed by a PNF-300 Profiling Natural Fluorometer sensor (Biospherical Instruments, Inc.). The limit of the euphotic zone corresponds to the depth where PAR is reduced to $1 \%$ of its surface value. Major nutrients and chlorophyll $a$ were measured as described in Raimbault et al. (2007).

For microplankton analysis, samples were collected at 14 stations from 5-280 m depth (Fig. 1). One hundred samples were preserved with acidified Lugol's solution and stored at $5^{\circ} \mathrm{C}$. A $500 \mathrm{ml}$ aliquot of the sample was concentrated by sedimentation in glass cylinders. During a six-day settling period, the top $450 \mathrm{ml}$ of the sample was slowly siphoned off with small-bore tubing. Fifty $\mathrm{ml}$ of the concentrate representing $500 \mathrm{ml}$ of whole water sample was used for settlement in a composite settling chamber. The entire chamber was scanned at $200 \times$ magnification under an Olympus inverted microscope equipped with a digital camera. The specimens were photographed at $400 \times$ magnification with the Olympus DP70-BSW software. This methodology is suitable for the counting and identification of organisms higher than $15 \mu \mathrm{m}$. Diatoms and dinoflagellates were 


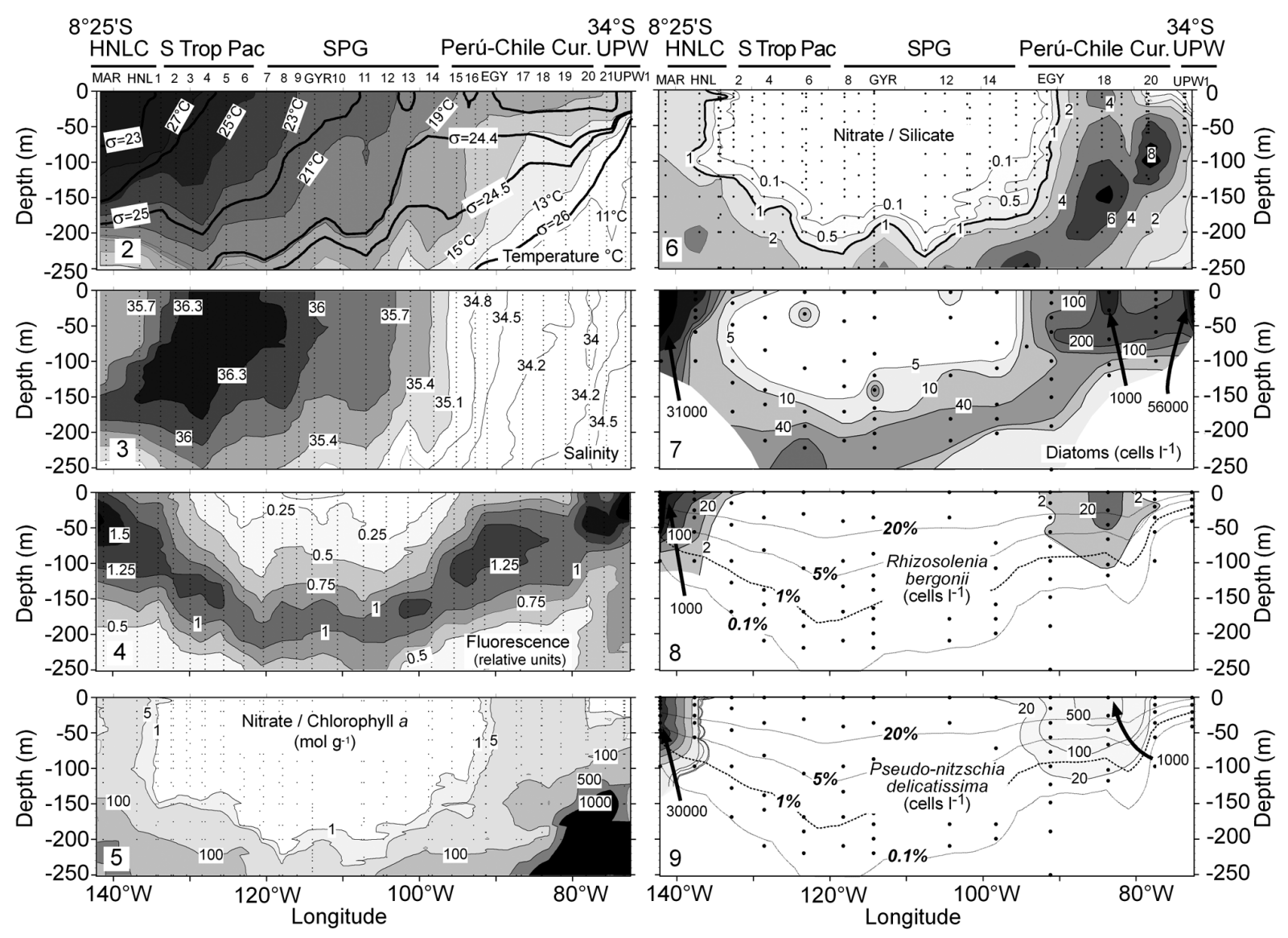

Fig. 2-9. Section plots of (2) temperature $\left({ }^{\circ} \mathrm{C}\right)$ and the isopycnals lines labelled in units of sigma-t (density-1000)(kg m $\left.{ }^{-3}\right)$ are shown. (3) Salinity. (4) Fluorescence (relative units). (5) Nitrate-chlorophyll $a$ ratio. (6) Nitrate-silicic acid ratio. (7) Abundance of diatoms. (8) Rhizosolenia bergonii and (9) Pseudo-nitzschia delicatissima (cells $1^{-1}$ ). The dashed lines represent the percentage of the surface irradiance.

well preserved, while most nanoplankton was lost due to preservation (i.e. coccolithophorids) or incomplete sedimentation. The organisms were identified to species level when possible.

\section{Results}

\subsection{Tropical HNLC Phytoplankton assemblage}

Waters surrounding the Marquesas Islands $\left(8^{\circ} \mathrm{S}, 141^{\circ} \mathrm{W}\right)$ under the influence of the South Equatorial Current constituted the southern border of HNLC waters of the Equatorial Upwelling Region. Salinity ranged from 35.5-35.7 and the surface temperature reached $27.9^{\circ} \mathrm{C}$. The vertical distribution of the fluorescence showed a wide maximum in the upper $50 \mathrm{~m}$ depth (Figs. 2-4). The concentrations of chlorophyll $a$ (Chl $a$ ) were about $0.3-0.5 \mu \mathrm{gl}^{-1}$ with values of the nitrateChl $a$ ratio higher than 5 (Fig. 5, Table 1).

Along $600 \mathrm{~km}$ between $8^{\circ} 23^{\prime} \mathrm{S}$ and $13^{\circ} 32^{\prime} \mathrm{S}$ extended the tropical HNLC-PA mainly represented small pennate diatom Pseudo-nitzschia delicatissima (30-80 $\mu \mathrm{m}$ in length,
2-3 $\mu \mathrm{m}$ in width) and the large centric diatom Rhizosole nia bergonii ( $>500 \mu \mathrm{m}$ in length). The microphytoplankton abundance reached up to 31000 cells $^{-1}$ in the upper $60 \mathrm{~m}$ depth of the surrounding waters the Marquesas Islands Archipelago (Fig. 7). Pseudo-nitzschia delicatissima dominated the HNLC-PA with more than $85 \%$ of the total abundance. This threadlike and lightly sicilified species was relatively homogeneously distributed in the upper $60 \mathrm{~m}$ depth, and often forming clusters of 10-20 cells (Figs. 10-11). Other abundant colonial congeneric species were Pseudonitzschia cf. subpacifica (Fig. 12) and Pseudo-nitzschia cf. pungens. Strongly elongated cells (up to $2000 \mu \mathrm{m}$ in length) of the pennate diatom Thalassiothrix longissima reached

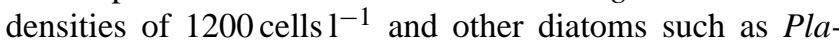
giotropis spp. and Haslea spp. were abundant. Rhizosolenia bergonii was dominant and reached an abundance of 1000 cells $^{-1}$ (maximum at 40-60 m depth, Figs. 13-14),

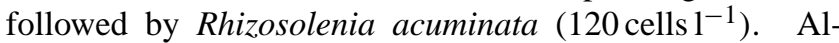
though the abundance of these diatoms was low compared to Pseudo-nitzschia spp., it should be taken into account that they are very large and thick, and contribute importantly to 
Table 1. Average values in the euphotic zone of chlorophyll $a\left(\mathrm{Chl} a, \mu \mathrm{gl} l^{-1}\right)$, nitrate $\left(\mathrm{NO}_{3}^{-}, \mu \mathrm{M}\right)$, phosphate $\left(\mathrm{PO}_{4}^{-3}, \mu \mathrm{M}\right)$, silicic acid $\left(\mathrm{Si}(\mathrm{OH})_{4}, \mu \mathrm{M}\right)$, nitrate-silicic acid ratio (N/Si), nitrate-phosphate ratio $(\mathrm{N} / \mathrm{P})$, abundance of diatoms $(>15 \mu \mathrm{m})$ and dinoflagellates $(>15 \mu \mathrm{m})$, units expressed as cells $1^{-1}$. Ze=depth of the euphotic zone calculated from the PNF-300 Profiling Natural Fluorometer sensor.

\begin{tabular}{|c|c|c|c|c|c|c|c|c|c|c|c|c|c|}
\hline \multicolumn{3}{|c|}{ HNLC-Marquesas } & \multicolumn{7}{|c|}{ Tropical and South Pacific Gyre } & \multicolumn{3}{|c|}{ Perú-Chile Current } & \multirow{2}{*}{$\begin{array}{r}\text { Upw. } \\
\text { UPW1 }\end{array}$} \\
\hline Station & MAR1 & HNL & 2 & 4 & 6 & 8 & GYR3 & 12 & 14 & EGY3 & 18 & 20 & \\
\hline $\mathrm{Ze}$ & 66 & 90 & 124 & 136 & 157 & 144 & 155 & 152 & 136 & 94 & 87 & 48 & 31 \\
\hline Chl $a$ & 0.46 & 0.24 & 0.17 & 0.12 & 0.03 & 0.07 & 0.10 & 0.01 & 0.09 & 0.19 & 0.20 & 0.53 & 1.10 \\
\hline $\mathrm{NO}_{3}^{-}$ & 1.48 & 1.52 & 0 & 0.04 & 0.02 & 0 & 0.01 & 0.01 & 0.01 & 0.30 & 4.08 & 2.18 & 2.38 \\
\hline $\mathrm{PO}_{4}^{-3}$ & 0.46 & 0.36 & 0.17 & 0.14 & 0.12 & 0.13 & 0.15 & 0.15 & 0.05 & 0.16 & 0.42 & 0.41 & 0.63 \\
\hline $\mathrm{Si}\left(\stackrel{\mathrm{OH}}{{ }_{4}}\right)_{4}$ & 1.17 & 1.27 & 0.59 & 1.04 & 1.15 & 1.16 & 0.85 & 0.86 & 0.83 & 0.99 & 0.57 & 0.68 & 2.56 \\
\hline $\mathrm{N} / \mathrm{Si}$ & 1.27 & 1.25 & 0 & 0.04 & 0.02 & 0 & 0.01 & 0.01 & 0.01 & 0.32 & 7.10 & 3.16 & 0.86 \\
\hline N/P & 3.23 & 4.34 & 0 & 0.30 & 0.19 & 0 & 0.06 & 0.04 & 0.13 & 1.61 & 9.73 & 4.64 & 3.62 \\
\hline DIATO & 25103 & 148 & 8 & 4 & 10 & 1 & 9 & 14 & 15 & 89 & 662 & 176 & 342548 \\
\hline DINO & 117 & 140 & 53 & 34 & 30 & 23 & 34 & 36 & 39 & 49 & 71 & 805 & 783 \\
\hline
\end{tabular}

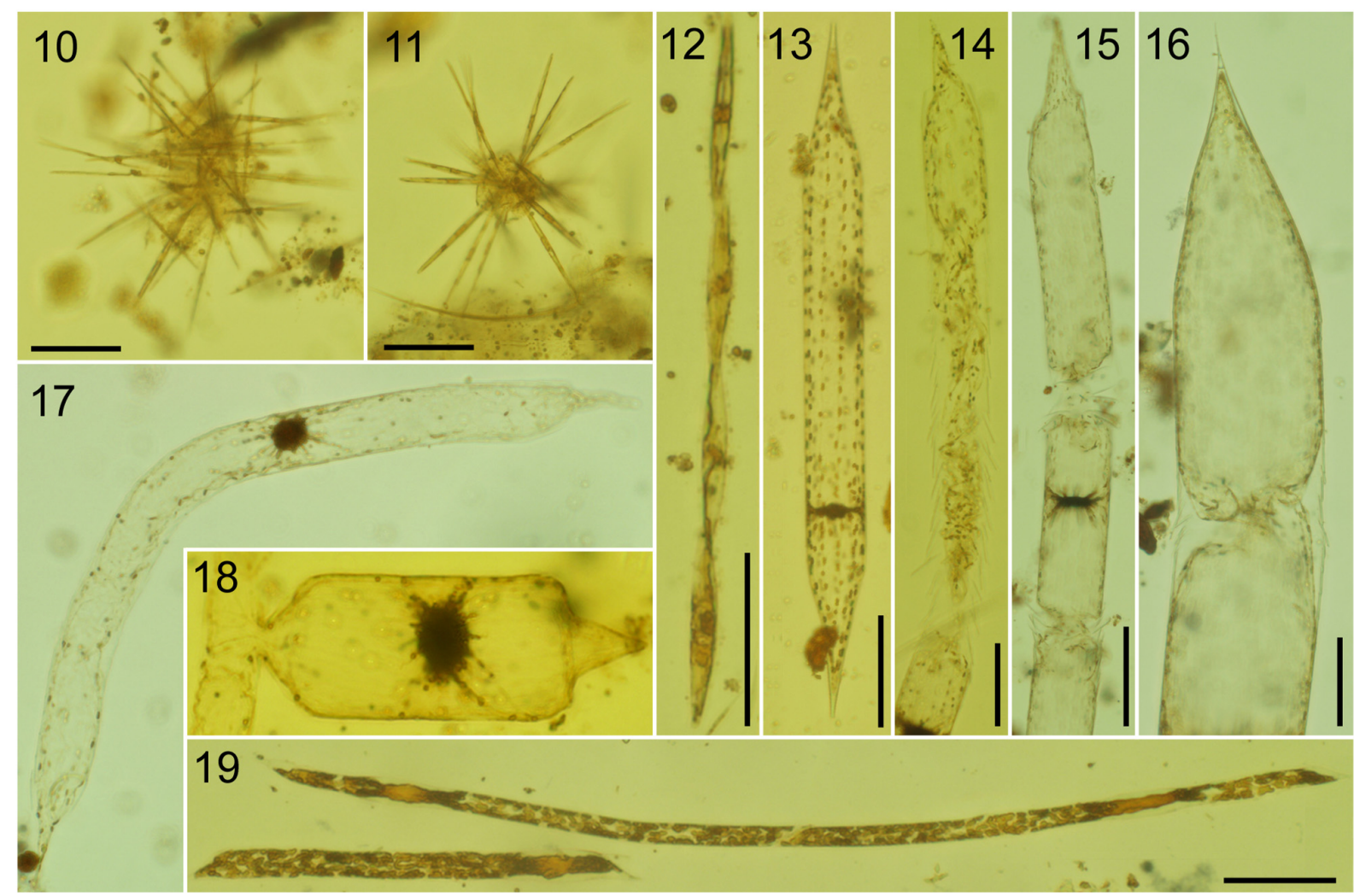

Fig. 10-19. Photomicrographs of microphytoplankton in the SE Pacific. (10-11) Clusters of Pseudo-nitzschia delicatissima, $20 \mathrm{~m}$ and $30 \mathrm{~m}$ depth. (12) Pseudo-nitzschia cf. subpacifica, $5 \mathrm{~m}$ depth. (13) "Normal" cell of Rhizosolenia bergonii, $60 \mathrm{~m}$ depth. (14) R. bergonii with fragmented frustule, $20 \mathrm{~m}$ depth. (10-14) $8^{\circ} 23^{\prime} \mathrm{S}$; $141^{\circ} 14^{\prime} \mathrm{W}$. (15) R. bergonii with fragmented frustule, $30 \mathrm{~m}$ depth. (16) Rhizosolenia acuminata, $5 \mathrm{~m}$ depth. (14-16) $9^{\circ} \mathrm{S}, 136^{\circ} 51^{\prime} \mathrm{W} ; 30$ and $5 \mathrm{~m}$ depth. (17) Curved cells of $R$. bergonii $\left(13^{\circ} 32^{\prime} \mathrm{S}, 132^{\circ} 07^{\prime} \mathrm{W}, 5 \mathrm{~m}\right.$ depth). (18) Auxospore of $R$. bergonii, $33^{\circ} 21^{\prime} \mathrm{S}, 78^{\circ} 06^{\prime} \mathrm{W}, 5 \mathrm{~m}$ depth. (19) Highly pigmented cells of Rhizosolenia sp. $\left(17^{\circ} 13^{\prime} \mathrm{S}, 127^{\circ} 58^{\prime} \mathrm{W}, 210 \mathrm{~m}\right.$ depth). Scale bar $=50 \mu \mathrm{m}$.

the phytoplankton biomass. Other common centric diatoms were Chaetoceros peruvianus (200 cells $1^{-1}$ near surface), Chaetoceros atlanticus var. neapolitanus, Planktoniella sol, Bacteriastrum cf. elongatum and Asteromphalus heptactis.
The dinoflagellates, ranging from $112-180$ cells $^{-1}$, showed a shallower distribution than the diatoms. The assemblage was rich on large autotrophic thecate species of the genus Ceratium (48-108 cells $1^{-1}$ ), mainly C. tripos, C. furca, 
C. massiliense, C. candelabrum, and unarmoured dinoflagellates such as Brachidinium capitatum f. Karenia papilionacea (12-24 cells $\left.1^{-1}\right)$, Pyrocystis spp. and the prasino-

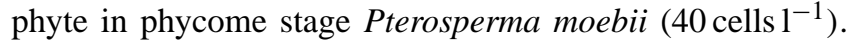
Among the heterotrophic species, dinoflagellates (Protoperidinium and Gyrodinium) and choanoflagellates were abundant (Table 2).

At a station located $480 \mathrm{~km}$ eastwards $\left(9^{\circ} \mathrm{S}, 136^{\circ} 51^{\prime} \mathrm{W}\right)$, diatom abundance decreased to $100-380$ cells $1^{-1}$ (Fig. 7) and was dominated by Rhizosolenia bergonii that accounted for half of diatoms (Fig. 8). The abundance of Pseudonitzschia delicatissima was low $\left(<140\right.$ cells $\left.1^{-1}\right)$ and the diatom clusters were not observed. The abundance of $R$. bergonii $\left(180\right.$ cells $^{-1}$ ) was less reduced than that of $P$. delicatissima in proportion to the previous station (Fig. 9). As occurred in the previous station, specimens of $R$. bergonii and $R$. acuminata showed the frustule anomalously fragmented in some concentric sections of the girdle (Figs. 1416).

The decrease of the diatom abundance was associated with an increase of dinoflagellates (100-230 cells $\left.1^{-1}\right)$. The autotrophic species of Ceratium were replaced by heterotrophic species such as $C$. teres. The abundance of diatomconsumers dinoflagellates (Protoperidinium spp.) decreased and smaller heterotrophic species such as Oxytoxum variabile and Podolompas spinifera were common.

To the south $\left(13^{\circ} 32^{\prime} \mathrm{S}, 132^{\circ} 07^{\prime} \mathrm{W}\right)$, the HNLC-PA was present with a very low abundance $\left(<50\right.$ cells $\left.1^{-1}\right)$ (Figs. 79). Pseudo-nitzschia delicatissima $\left(20\right.$ cells $\left.^{-1}\right)$, Planktoniella sol, a few specimens of $R$. bergonii, and Thalassiothrix sp. were encountered in the surface layer. Near the bottom of the euphotic (100-150 m depth, see Fig. 3), the salty South Tropical Pacific Waters coincided with highly pigmented specimens of Rhizosolenia cf. imbricata (Fig. 19), and Nitzschia bicapitata species complex at $170 \mathrm{~m}$ depth. The abundance of dinoflagellates ranged from 40 110 cells $1^{-1}$.

\subsection{Temperate HNLC phytoplankton assemblage}

Between $30^{\circ} \mathrm{S}$ and $32^{\circ} \mathrm{S}$ extended a subtropical front characterized by decrease of the salinity and temperature, and the shoaling of the isopicne of $1025.4 \mathrm{Kg} \mathrm{m}^{-3}$. The isohalines between 35.3 and 34.6 were vertically distributed in the upper $200 \mathrm{~m}$. From 50-200 $\mathrm{m}$ depth, the isotherms were vertically distributed decreasing between $18^{\circ} \mathrm{C}$ and $16^{\circ} \mathrm{C}$ (Figs. 2-3). To the east, along the salinity frontal region the fluorescence increased and shoaled between $120 \mathrm{~m}$ and 70 $80 \mathrm{~m}$ depth (Fig. 4). The nitrate-Chl $a$ ratio showed values higher than 5 (Fig. 5).

The Perú-Chile Current is a width equatorward coldwater current. The isopicne of $1025.4 \mathrm{~kg} \mathrm{~m}^{-3}$ (Fig. 2) and the depth of the fluorescence maximum were horizontally distributed (Fig. 4). The PCC, delimited by isohaline of 34.3, was associated with fresher waters, especially between $78^{\circ} \mathrm{W}$ and $75^{\circ} \mathrm{W}$ (salinity $~ 34.1$ ). Near the Juan Fernández Archipelago (650 km off Chile) the salinity decreased to 33.9 and isotherm of $11^{\circ} \mathrm{C}$ shoaled (Figs. 2-3). This fresher water mass found in the surface may correspond to the Eastern South Pacific Intermediate Water as defined by Schneider et al. (2003). In these conditions, the fluorescence increased and shoaled (Fig. 4). The ADCP measurements showed a northwards component between $92^{\circ} \mathrm{W}$ and $84^{\circ} \mathrm{W}$, while the current was weak and often reverse towards the coasts of Chile.

Large phytoplankton was nearly absent in the upper $120 \mathrm{~m}$ of the vast region of the SPG with very deep maximum composed of Nitzschia bicapitata species complex, Nitzschia spp. and other small pennate diatoms. The large phytoplankton in the upper layer reappeared towards the western PerúChile Current. The surface phytoplankton assemblage found in the PCC coincided to that observed in the central tropical Pacific. About $2000 \mathrm{~km}$ off Chile under the influence of PCC $\left(31^{\circ} 52^{\prime} \mathrm{S}, 91^{\circ} 24^{\prime} \mathrm{W}\right)$ the phytoplankton was constituted of the typical HNLC-PA in surface and deeper the diatom assemblage found in the oligotrophic waters of the SPG. In upper $100 \mathrm{~m}$, the diatom abundance was 50-196 cells $1^{-1}$ composed of Pseudo-nitzschia delicatissima $\left(160\right.$ cells $\left.^{-1}\right)$, Rhizosolenia bergonii $\left(8\right.$ cells $\left.1^{-1}\right), R$. acuminata $\left(6\right.$ cells $\left.1^{-1}\right)$, and a few specimens of Plagiotropis sp. and Thalassiothrix sp. Deeper than $100 \mathrm{~m}$ depth and parallel to the shoaling of the bottom of the euphotic zone, the abundance of the diatoms was low and composed of Dactyliosolen sp. (80-150 $\mathrm{m}$ depth), Chaetoceros atlanticus var. neapolitanus and Thalassiosira sp. (160 cells $^{-1}$ at $80 \mathrm{~m}$ depth) (Fig. 7).

In the upper $60 \mathrm{~m}$ depth, the abundance of dinoflagellates ranged from 54-168 cells $1^{-1}$. The autotrophic dinoflagellates Gonyaulax polygramma $\left(60\right.$ cells $\left.^{-1}\right)$, Ceratium fusus $\left(40\right.$ cells $\left.^{-1}\right)$, $C$. furca were responsible of the surface maximum. Other autotrophic species were C. tripos, C. azoricum, C. canderabrum, C. kofoidii as observed in the warm HNLC region and brachidiniaceans. A diverse assemblage of heterotrophic dinoflagellates of more eutrophic conditions was present (Table 2).

In temperate waters $\sim 1300 \mathrm{~km}$ off Chile $\left(32^{\circ} 42^{\prime} \mathrm{S}\right.$, $84^{\circ} 04^{\prime} \mathrm{W}$ ) the nitrate-silicic acid ratio was higher than 4 in the euphotic zone, with a maximum of 6 in the surface (Fig. 6, Table 1). The diatom assemblage was similar to that in HNLC conditions near the Marquesas Islands Archipelago. The diatom abundance showed values up to 1200 cells $^{-1}$ in upper layer (Fig. 7) dom-

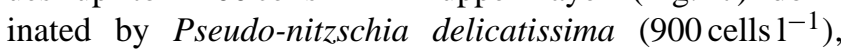
Pseudo-nitzschia cf. subpacifica $\left(120\right.$ cells $\left.1^{-1}\right)$, Rhizosole-

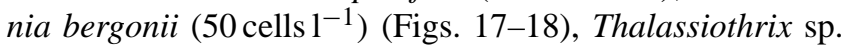
$\left(40\right.$ cells $\left.^{-1}\right)$, Planktoniella sol $\left(16\right.$ cells $\left.1^{-1}\right)$ and Asteromphalus heptactis $\left(20\right.$ cells $\left.^{-1}\right)$. Dactyliosolen sp. and Chaetoceros atlanticus var. neapolitanus were encountered below the surface phytoplankton assemblage. The abundance of dinoflagellates was low $\left(40-78\right.$ cells $\left.^{-1}\right)$, mainly composed of Pronoctiluca pelagica f. spinifera 
Table 2. List of diatoms, dinoflagellates $(>15 \mu \mathrm{m})$ and large flagellates during the BIOSOPE cruise. HNLC=High-Nutrient Low-Chlorophyll region near the Marquesas Islands Archipelago; SPG=Tropical and South Pacific Gyre; PCC=Perú-Chile Current; UPW=Coastal upwelling off Concepción.

\begin{tabular}{|c|c|c|c|c|}
\hline Diatom taxa & HNLC & SPG & $\mathrm{PCC}$ & UPW \\
\hline Asterionellopsis glacialis (Castracane) Round & & & & + \\
\hline Asterolampra marylandica Ehrenberg & & + & & \\
\hline Asteromphalus flabellatus (Brébisson) Greville & + & + & + & + \\
\hline Bacillaria cf. paxillifera (O.F. Müller) Hendey & + & & & \\
\hline Bacteriastrum cf. comosum Pavillard & + & & + & \\
\hline Bacteriastrum cf. furcatum Shadbolt & + & & & \\
\hline Bacteriastrum sp1 & & + & + & \\
\hline Bacteriastrum sp2 & & & & + \\
\hline Bacteriastrum sp3 & & + & & \\
\hline Chaetoceros affinis Lauder & & & & + \\
\hline Chaetocero atlanticus var. neapolitanus (Schröder) Hustedt & + & + & + & \\
\hline Chaetoceros compressus Lauder & & & & + \\
\hline Chaetoceros curvisetus Cleve & & & & + \\
\hline Chaetoceros dadayi Pavillard & + & + & + & \\
\hline Chaetoceros cf. diadema (Ehrenberg) Gran & & & & + \\
\hline Chaetoceros didymus Ehrenberg & & & & + \\
\hline Chaetoceros cf. diversus Cleve & & + & & \\
\hline Chaetoceros laciniosus Schütt & & & & + \\
\hline Chaetoceros lauderi Ralfs & & & & + \\
\hline Chaetoceros lorenzianus Grunow & + & & & + \\
\hline Chaetoceros peruvianus Brightwell & + & + & + & \\
\hline Chaetoceros radicans Schütt & & & & + \\
\hline Chaetoceros rostratus Lauder & & & & + \\
\hline Chaetoceros cf. tenuissimus Meunier & & & & + \\
\hline Chaetoceros tetrastichon Cleve & + & + & & \\
\hline Chaetoceros sp1 & & + & & \\
\hline Chaetoceros $\mathrm{sp} 2$ & & + & & \\
\hline Chaetoceros $\mathrm{sp} 3$ & + & + & & \\
\hline Chaetoceros sp4 & & + & & \\
\hline Chaetoceros sp5 & & + & & \\
\hline Corethron cf. criophilum Castracane & & & & + \\
\hline Coscinodiscus sp. & & + & & \\
\hline Dactyliosolen sp. & & + & + & \\
\hline Dactyliosolen fragilissimus (Bergon) Hasle & & & & + \\
\hline Detonula pumila (Castracane) Schütt & & & & + \\
\hline Eucampia cornuta (Cleve) Grunow & & & & + \\
\hline Eucampia zodiacus Ehrenberg & & & & + \\
\hline Fragilariopsis doliolus (Wallich) Medlin et Sims & + & & & \\
\hline Guinardia delicatula (Cleve) Hasle & & & & + \\
\hline Guinardia flaccida (Castracane) Peragallo & & & & + \\
\hline Guinardia striata (Stolterfoth) Hasle & & & & + \\
\hline Grossleriella tropica Schütt & & + & & \\
\hline Hemiaulus hauckii Grunow & & + & & \\
\hline Hemidiscus cuneiformis Wallich & + & & & \\
\hline Lauderia annulata Cleve & & & & + \\
\hline Leptocylindrus danicus Cleve & & & & + \\
\hline Neostreptotheca torta f. triangularis Stosch & & + & & \\
\hline Nitzschia bicapitata species complex & + & + & + & \\
\hline Nitzschia longissima (Brébisson) Ralfs & + & & + & + \\
\hline
\end{tabular}


Table 2. Continued.

\begin{tabular}{|c|c|c|c|c|}
\hline Diatom taxa & HNLC & SPG & PCC & UPW \\
\hline Nitzschia sp. (?braarudii) & & + & + & \\
\hline Odontella cf. longicrucis (Greville) Hoban & & & & + \\
\hline Plagiotropis sp. & + & + & + & \\
\hline Plagiotropis sp. (?lepidoptera) & + & + & + & \\
\hline \multicolumn{5}{|l|}{ Planktoniella sol (Wallich) Schütt } \\
\hline Proboscia alata (Brightwell) Sundström & + & + & + & + \\
\hline Pseudotriceratium cinnamomeum (Greville) Grunow & & + & & \\
\hline Pseudo-nitzschia delicatissima (Cleve) Heiden sensu Hasle 1960 & + & + & + & \\
\hline Pseudo-nitzschia cf. pungens (Grunow ex Cleve) Hasle & + & & + & \\
\hline Pseudo-nitzschia cf. subpacifica (Hasle) Hasle & + & & + & + \\
\hline Pseudo-nitzschia sp1 & & + & & \\
\hline Pseudo-nitzschia $\mathrm{sp} 2$ & & & & + \\
\hline Pseudo-nitzschia sp3 & & & & + \\
\hline cf. Pseudoeunotia sp. & & + & + & \\
\hline \multicolumn{5}{|l|}{ Rhizosolenia acuminata (Peragallo) Gran } \\
\hline Rhizosolenia bergonii Peragallo & + & + & + & \\
\hline Rhizosolenia cf. castracanei Peragallo & & + & & \\
\hline Rhizosolenia clevei var. communis Sundström & & + & & \\
\hline Rhizosolenia cf. imbricata Brightwell & & + & & + \\
\hline Rhizosolenia cf. imbricata var. shrubsolei (Cleve) Schröder & & + & & + \\
\hline Rhizosolenia setigera Brightwell & & & & + \\
\hline Rhizosolenia styliformis Brightwell & + & + & & \\
\hline Skeletonema sp. & & & & + \\
\hline Thalassionema nitzschioides (Grunow) Mereschkowsky & & + & & + \\
\hline Thalassionema cf. pseudonitzschioides (Schuette et Schrader) Hasle & & + & & + \\
\hline Thalassionema elegans Hustedt & + & + & & \\
\hline Thalassionema sp. & & + & & \\
\hline Thalassiosira anguste-lineata (A. Schmidt) Fryxell et Hasle & & & & + \\
\hline Thalassiosira cf. decipiens (Grunow) Jørgensen & & & & + \\
\hline Thalassiosira cf. oestrupii (Ostenfeld) Hasle & + & & + & \\
\hline Thalassiosira subtilis (Ostenfeld) Gran & + & & + & + \\
\hline Thalassiosira sp. & & & + & \\
\hline Thalassiothrix longissima Cleve et Grunow & + & + & + & + \\
\hline Thalassiothrix cf. heteromorpha Karsten & + & + & + & \\
\hline \multicolumn{5}{|l|}{ Dinoflagellate taxa } \\
\hline cf. Amphidoma caudata Halldal & + & & & \\
\hline Amphisolenia bidentata Schröder & & + & + & \\
\hline Amphisolenia globifera Stein & + & + & + & \\
\hline cf. Blephaerocysta sp. & + & + & + & \\
\hline Brachidinium capitatum F.J.R. Taylor f. Asterodinium & + & + & + & + \\
\hline Brachidinium capitatum F.J.R. Taylor f. Brachidinium & + & + & + & \\
\hline Brachidinium capitatum F.J.R. Taylor f. Karenia papilionacea & + & + & + & + \\
\hline Brachidinium capitatum F.J.R. Taylor f. Microceratium & & + & & \\
\hline Ceratium arietinum Cleve & & + & & \\
\hline Ceratium azoricum Cleve & + & & + & + \\
\hline Ceratium belone Cleve & & & + & \\
\hline Ceratium breve (Ostenfeld et Schmidt) Schröder & + & & & \\
\hline Ceratium candelabrum (Ehrenberg) Stein & + & & + & \\
\hline Ceratium carnegiei Graham et Bronikowsky & + & & + & \\
\hline Ceratium carriense Gourret & + & + & + & \\
\hline Ceratium cf. lineatum (Ehrenberg) Cleve & + & & & \\
\hline
\end{tabular}


Table 2. Continued.

\begin{tabular}{|c|c|c|c|c|}
\hline Dinoflagellate taxa & HNLC & SPG & PCC & UPW \\
\hline Ceratium contortum (Gourret) Cleve & & & + & \\
\hline Ceratium declinatum (Karsten) Jørgensen & + & + & & \\
\hline Ceratium divaricatum (Lemmermann) Kofoid var. divaricatum & & & + & \\
\hline Ceratium divaricatum var. balechii (Meave, Okolodkov et Zamudio) Hernández-Becerril & + & & & + \\
\hline Ceratium extensum (Gourret) Cleve & & + & + & \\
\hline Ceratium falcatum (Kofoid) Jørgensen & + & & & \\
\hline Ceratium furca (Ehrenberg) Claparède et Lachmann & + & + & + & + \\
\hline Ceratium fusus (Ehrenberg) Dujardin & + & + & + & + \\
\hline Ceratium gibberum Gourret & + & + & + & \\
\hline Ceratium horridum (Cleve) Gran & & & & + \\
\hline Ceratium kofoidii Jørgensen & + & + & + & \\
\hline Ceratium lanceolatum Kofoid & & & + & \\
\hline Ceratium limulus Gourret & & & + & \\
\hline Ceratium massiliense (Gourret) Jørgensen & + & + & + & \\
\hline Ceratium pentagonum Gourret & + & + & + & + \\
\hline Ceratium platycorne Daday & & + & & \\
\hline Ceratium praeolongum (Lemmermann) Kofoid & & & + & \\
\hline Ceratium pulchellum Schröder & & & & + \\
\hline Ceratium symmetricum Pavillard & & + & + & \\
\hline Ceratium teres Kofoid & + & + & & \\
\hline Ceratium trichoceros (Ehrenberg) Kofoid & + & + & + & + \\
\hline Ceratium tripos (O.F. Müller) Nitzsch & + & + & + & + \\
\hline Ceratocorys bipes (Cleve) Kofoid & & & + & \\
\hline Ceratocorys horrida Stein & & & + & \\
\hline Citharistes sp. & + & & & \\
\hline Cladopyxis brachiolata Stein & + & + & + & \\
\hline Cladopyxis sp. & + & + & + & + \\
\hline Cochlodinium sp. & & + & + & + \\
\hline Craspedotella pileolus Kofoid & & + & & \\
\hline Craspedotella sp. & + & + & + & + \\
\hline Dicroerisma psilonereiella F.J.R. Taylor et Cattell & + & + & + & \\
\hline Dinophysis acuminata Claparède et Lachmann & & & & + \\
\hline Dinophysis cf. swezyae Kofoid et Skogsberg & & + & & \\
\hline Dinophysis fortii Pavillard & & & + & \\
\hline Dinophysis mucronata (Kofoid et Skogsberg) Sournia & + & & + & \\
\hline Dinophysis schuettii Murray et Whitting & & + & + & \\
\hline Erythropsidinium agile (Hertwig) P.C. Silva & + & + & + & + \\
\hline cf. Gonyaulax birostris Stein & & & + & \\
\hline Gonyaulax polygramma Stein & + & + & + & \\
\hline Gymnodinium fusus Schütt/Gyrodinium falcatum Kofoid et Swezy & + & + & + & \\
\hline Gymnodinium sp1 & & & & + \\
\hline Gymnodinium sp2 & & + & & \\
\hline Gyrodinium spp. & + & & & + \\
\hline Gyrodinium spirale (Bergh) Kofoid et Swezy & + & + & + & + \\
\hline Histioneis cf. crateriformis Stein & & + & + & \\
\hline Histioneis cf. pulchra Kofoid & & & + & \\
\hline Histioneis cf. striata Kofoid et Michener & & & + & \\
\hline Histioneis cleaveri Rampi & + & + & & \\
\hline Histioneis hyalina Kofoid et Michener & + & & & \\
\hline Histioneis joergensenii Schiller & & + & & \\
\hline Histioneis longicollis Kofoid & & & + & \\
\hline
\end{tabular}


Table 2. Continued.

\begin{tabular}{|c|c|c|c|c|}
\hline Dinoflagellate taxa & HNLC & SPG & PCC & UPW \\
\hline cf. Histiophysis sp. & & & + & \\
\hline Karenia cf. bicuneiformis Botes, Sym et Pitcher & & + & & \\
\hline Karenia cf. mikimotoi (Miyake et Kominami ex Oda) G. Hansen et Moestrup & + & & + & \\
\hline Karenia spp. & + & + & + & + \\
\hline Kofoidinium pavillardii J. Cachon et M. Cachon & + & + & + & \\
\hline Kofoidinium sp. & + & + & + & \\
\hline Kofoidinium velelloides Pavillard & + & + & + & \\
\hline Leptodiscus medusoides Hertwig & & & + & \\
\hline cf. Metaphalacroma skogsbergii Tai in Tai et Skogsberg & & & + & \\
\hline Nematodinium sp. & + & + & + & \\
\hline Ornithocercus magnificus Stein & & + & + & \\
\hline Ornithocercus quadratus Schütt & + & + & + & \\
\hline Ornithocercus sp. & + & + & & \\
\hline Oxytoxum cf. crassum Schiller & + & + & + & \\
\hline Oxytoxum cf. frenguellii Rampi & & & + & \\
\hline Oxytoxum cf. laticeps Schiller & + & + & & + \\
\hline Oxytoxum cf. longum Schiller & & + & + & \\
\hline Oxytoxum challengeroides Kofoid & & + & & \\
\hline Oxytoxum constrictum (Stein) Bütschli & & + & + & \\
\hline Oxytoxum curvatum (Kofoid) Kofoid & + & + & + & \\
\hline Oxytoxum curvicaudatum Kofoid & & + & + & \\
\hline Oxytoxum diploconus Stein & + & + & + & \\
\hline Oxytoxum longiceps Schiller & + & + & + & \\
\hline Oxytoxum scolopax Stein & + & + & + & + \\
\hline Oxytoxum sp1 & & & + & \\
\hline Oxytoxum $\mathrm{sp} 2$ & + & & & \\
\hline Oxytoxum sp3 & & + & & \\
\hline Oxytoxum sp4 & & & + & \\
\hline Oxytoxum tesselatum (Stein) Schütt & + & + & + & \\
\hline Oxytoxum variabile Schiller & + & + & + & \\
\hline Phalacroma rotundatum (Claparède et Lachmann) Kofoid et Michener & & & + & \\
\hline Phalacroma sp1 & + & + & + & \\
\hline Phalacroma $\mathrm{sp} 2$ & + & & & \\
\hline Phalacroma sp3 & & + & & \\
\hline Phalacroma sp4 & & + & & \\
\hline Phalacroma sp5 & & + & + & \\
\hline Podolampas bipes Stein & + & + & + & \\
\hline Podolampas elegans Schütt & & + & + & + \\
\hline Podolampas palmipes Stein & + & + & + & \\
\hline Podolampas sp. & + & & & \\
\hline Podolampas spinifera Okamura & + & + & + & + \\
\hline Polykrikos cf. schwartzii Bütschli & & + & + & + \\
\hline cf. Pomatodinium impatiens J. Cachon et Cachon-Enjumet & + & & + & \\
\hline Pronoctiluca pelagica Fabre-Domergue & + & + & & \\
\hline Pronoctiluca pelagica Fabre-Domergue f. spinifera & + & + & + & + \\
\hline Prorocentrum cf. compressum (Bailey) Abé ex Dodge & + & & & \\
\hline Prorocentrum dentatum Schiller & + & + & & \\
\hline Prorocentrum rostratum Stein & + & + & + & \\
\hline Prorocentrum sp. & & & + & \\
\hline Protoperidinium cf. conicum (Gran) Balech & + & & & \\
\hline Protoperidinium cf. curtipes (Jørgensen) Balech & + & & + & + \\
\hline
\end{tabular}


Table 2. Continued.

\begin{tabular}{|c|c|c|c|c|}
\hline Dinoflagellate taxa & HNLC & SPG & PCC & UPW \\
\hline Protoperidinium cf. depressum (Bailey) Balech & & & & + \\
\hline Protoperidinium cf. divergens (Ehrenberg) Balech & + & & & \\
\hline Protoperidinium cf. murrayi (Kofoid) Hernández-Becerril & + & & + & \\
\hline Protoperidinium cf. obtusum (Karsten) Parke et Dodge & + & & & \\
\hline Protoperidinium cf. ovatum Pouchet & & + & & \\
\hline Protoperidinium cf. steinii (Jørgensen) Balech & + & + & + & + \\
\hline Protoperidinium sp. & + & & & + \\
\hline Ptychodiscus noctiluca Stein & + & & + & \\
\hline Pyrocystis fusiformis (Wyville Thomson ex Haeckel) Blackman & + & & & \\
\hline Pyrocystis lunula (Schütt) Schütt & + & + & + & \\
\hline Pyrocystis noctiluca J. Murray ex Haeckel & + & + & + & \\
\hline cf. Pyrocystis obtusa Pavillard & + & & + & \\
\hline Pyrophacus steinii (Schiller) Wall et Dale & & & + & + \\
\hline Scaphodinium mirabile Margalef & + & + & + & \\
\hline Scaphodinium sp. & & + & & \\
\hline Scrippsiella sp. & & + & & + \\
\hline Spatulodinium pseudonoctiluca (Pouchet) J. Cachon et $\mathrm{M}$. Cachon & & & + & + \\
\hline Spatulodinium sp1 & & + & + & \\
\hline Spatulodinium sp2 & & + & & \\
\hline cf. Spiraulax jolliffei (Murray et Whitting) Kofoid & & + & + & \\
\hline Torodinium teredo (Pouchet) Kofoid et Swezy & + & + & + & + \\
\hline \multicolumn{5}{|l|}{ Large flagellate taxa } \\
\hline Dictyocha fibula Ehrenberg & + & & + & + \\
\hline Pterosperma moebii (Jørgensen) Ostenfeld & + & + & + & \\
\hline Pterosperma cuboides Gaarder & & + & & \\
\hline Halosphaera viridis Schmitz & & + & & \\
\hline Solenicola setigera Pavillard & + & + & + & + \\
\hline
\end{tabular}

(40 cells $\left.1^{-1}\right)$, Ptychodiscus noctiluca, Ornithocercus magnificus, Phalacroma rotundatum, Gyrodinium falcatum, Gonyaulax polygramma $\left(12\right.$ cells $\left.1^{-1}\right)$, Ceratium fusus, and Dinophysis fortii $\left(8\right.$ cells $\left.^{-1}\right)$ (Table 2).

About $650 \mathrm{~km}$ off Chile, a sampling station $\left(33^{\circ} 21^{\prime} \mathrm{S}\right.$, $78^{\circ} 06^{\prime} \mathrm{W}$ ) located $30 \mathrm{~km}$ north from the Robinson Crusoe Island, Juan Fernández Archipelago, was visited. The salinity reached a minimum of 33.9 in the surface. The ADCP measurements showed an eastwards drift and the surface warm waters run above the entrainment of fresher waters. This - surface stratified upwelling - resulted on a red tide of dinoflagellates with a surface abundance of 4400 cells $^{-1}$. The main component of the red tide was the non-toxic autotrophic dinoflagellate Gonyaulax polygramma that reached 3800 cells $1^{-1}$ at $5 \mathrm{~m}$ depth, 530 cells $1^{-1}$ at $25 \mathrm{~m}$, and nearly disappeared below this depth. Other autotrophic dinoflagellates were Ceratium azoricum $\left(90\right.$ cells $\left.^{-1}\right), C$. massiliense, C. gibberum, C. limulus, Ptychodiscus noctiluca and brachidiniaceans. The heterotrophic dinoflagellates reached a high abundance, mainly composed of Protoperidinium cf. curtipes $\left(120\right.$ cells $\left.1^{-1}\right)$, Ornithocercus quadratus, Gyrodinium spirale in the upper 5-15 m depth as well as kofoidiniaceans (Kofoidinium and Spatulodinium). Dinoflagellates such as Oxytoxum longum and Pronoctiluca pelagica f. spinifera were encountered at mid-depths. The abundance of diatoms ranged from 100-600 cells $1^{-1}$ (Fig. 7) with Pseudo-nitzschia delicatissima, Rhizosolenia bergonii $\left(4\right.$ cells $^{-1}$ ), and Pseudo-nitzschia cf. subpacifica in the surface layer, Thalassiosira sp. $\left(180\right.$ cells $\left.\mathrm{l}^{-1}\right)$ at $25 \mathrm{~m}$ depth and Dactyliosolen sp. at $60 \mathrm{~m}$ depth were encountered (Table 2).

\section{Discussion}

4.1 High Nutrient-Low Chlorophyll phytoplankton assemblages

The HNLC-PA in the southeast Pacific was dominated by the lightly silicified diatoms Pseudo-nitzschia delicatissima and Rhizosolenia bergonii that were encountered in the Marquesas Islands Archipelago at $8^{\circ} \mathrm{S}$ with a surface temperature of $28^{\circ} \mathrm{C}$ and salinity 35.5 , and between 2000 and $600 \mathrm{~km}$ off the Chilean coast at $33^{\circ} \mathrm{S}$ with a surface temperature of $17^{\circ} \mathrm{C}$ and salinity of 34 (Figs. 2-3, 8-9). The western side of the PCC did not appear in the literature as one of the HNLC regions of the world's oceans. The HNLC-PA showed scarce differences in these geographically distant regions. Only a few tropical species such as the dinoflagellate genus 
Citharistes was absent in temperate waters (Table 2). HNLC diatoms such as Planktoniella sol or Rhizosolenia bergonii are commonly reported along the South American coastal waters associated with warm waters especially during the El Niño conditions (Avaria and Muñoz, 1987; Rodríguez et al., 1996). Based on a single survey and with a paucity of information in the literature in offshore waters in the region, the present study does not allow to establish the occurrence of the HNLC-PA in the western PCC as a permanent feature. In the SE Pacific the HNLC-PA was encountered in two regions of contrasted temperature and salinity values. Other trophic or ecological factor(s) may determinate the occurrence of the HNLC-PA in these two distant regions.

\subsubsection{Grazing and the HNLC-PA}

One of the explanations for the HNLC paradox is that phytoplankton populations are strictly controlled by the zooplankton (Frost and Franzen, 1992; Leising et al., 2003). In the equatorial Pacific, Roman and Gauzens (1997) suggested that the scarce copepods mainly grazed on protozoa. Landry et al. (1995) reported that grazing by microzooplankton dominates phytoplankton losses, and that large diatoms are not easily exploited by protistan consumers (ciliates, dinoflagellates, etc). The microscope observations in the present study showed a very low abundance of copepods, although the scarce volume examined $(0.5 \mathrm{l})$ were insufficient to provide a statistically valid estimation of the early stages of copepods abundance. The ciliates and heterotrophic dinoflagellates (Gyrodinium spp. and Protoperidinium spp.) were abundant.

The two most successful species in the HNLC regions of the SE Pacific, Pseudo-nitzschia delicatissima and Rhizosolenia bergonii, seems to develop two different antigrazing strategies. Pseudo-nitzschia delicatissima, and occasionally Nitzschia longissima increased theirs sizes by forming clumps or "ball of needles" of $\sim 100 \mu \mathrm{m}$ in diameter disposed in all directions (Figs. 10-11). This diatom behavior was already described by Hasle (1960) and Buck and Chavez (1994). The aggregation of these diatoms suggests an anti-grazing adaptation in an environment dominated by micrograzers. On the other hand, centric diatoms such as $R h i$ zosolenia bergonii and $R$. acuminata reached sizes $>500 \mu \mathrm{m}$ in length, being far of the prey size spectra of most micrograzers.

Other diatom species are not exclusive of the HNLC conditions, although they are favoured in these eutrophic conditions. Diatoms showed strongly elongated frustules or extensions. For example Thalassiothrix longissima is thin, but reached $2000 \mu \mathrm{m}$ in length. Planktoniella sol secretes a large hyaline ring that increases its size. Chaetoceros peruvianus is a uni-cellular species with long spine-bearing setae. Colonial species such as Chaetoceros atlanticus var. neapolianus and Bacteriastrum cf. elongatum showed a similar strategy with long setae.
Beyond the size increase or aggregation of the diatoms as possible response to small grazers, it should be taken into account that the assemblage was dominated by Pseudonitzschia, a genus that contains numerous species able to produce the domoic acid. Reactive aldehydes produced by diatoms have been reported to reduce the fecundity and egg hatching success of copepods (Ianora et al., 2003). The effects of the compounds produced by diatoms such as the domoic acid, if produced, on the abundance of copepods in the HNLC region need further research.

\subsubsection{Lightly silicified diatoms near the surface?}

A rigid and thick frustule of diatoms is considered to be the main anti-grazing strategy against copepods. If copepods are not abundant in HNLC conditions, lightly silicified diatoms may be favoured. The dominant diatoms of the HNLC regions are characterized by thin frustules and the lack siliceous ornamentation requiring a superfluous silicon uptake. Pseudo-nitzschia delicatissima and $R$. bergonii easily dissolved their lightly silicified frustules and these diatoms are nearly absent from the sediments (Kolbe, 1954). At first sight, a lightly silicified frustule may be a response to an environment where the silicic acid bioavailability is low. In the present study the HNLC-PA appeared in two regions with different hydrographical conditions, but apparent similar trophic conditions. The P. delicatisima-R. bergonii assemblage (Figs. 8-9) was associated with a nitrate-silicic acid ratio that ranged from 1-3 in the euphotic zone of the two HNLC regions (Fig. 6, Table 1). The elemental composition of the diatoms is characterized by nitrogen-silicic acid ratio of 1 (Brzezinski, 1985). It can be expected that the diatoms uptake the nutrients from the surrounding waters with a similar ratio. Values of the nitrate-silicic acid ratio higher than 1, may imply a silicon limitation for the diatom growth. However, the nutrient limitation is a complex issue, and only the nutrient ratios are not suitable to infer limitations. Based on silicic acid uptake measurements, Leynaert et al. (2001) reported direct evidence that the diatoms are Si-limited in the central equatorial Pacific.

It is evident that the diatoms must be adapted to optimize the silicic acid utilization. Large frustules such as those in $R$. bergonii and $R$. acuminata may allow to escape of most of micrograzers. The balance between large frustules avoiding the micrograzers and a thin frustule that allowed to economize the scarce silicic acid available may result in deficiencies in the formation and maintenance of the frustule. In the present study, specimens of Rhizosolenia bergonii and $R$. acuminata were curved instead of the usual rigid frustules and sections of the frustules appeared anomalously fragmented (Figs. 12-15). This phenomenon did not occur with other diatoms and the plasmalemma of the diatom appeared retracted in the regions where the frustule is fragmented. The portion of the frustule closer to the diatom nucleus did not usually appear fragmented (Figs. 14-15). Consequently this 
phenomenon of fragmentation of the frustule occurred before the fixation of the samples, discarding an artifact due to the fixation methodology. This is evidence that the silicic acid is an element limiting the large diatoms $R$. bergonii and $R$. acuminata in the HNLC regions of the South Pacific Ocean.

The possible deficiency of silicic acid may determinate the presence of other phytoplankton components of the HNLCPA. Chavez (1989) included the absence of neritic bloomforming diatoms among the factors to explain the HNLC paradox. Although colonial diatoms such as Chaetoceros lorenzianus were observed in the present study, well silicified neritic diatoms (i.e. Detonula, Leptocylindrus, Guinardia, etc.) that are dominant in mesotrophic coastal waters were absent in the HNLC regions. The typical neritic diatoms may be less competitive in the HNLC regions versus the lightly silicificed diatoms. For example Planktoniella sol has developed an organic ring-like wing that does not contain silica. Beyond the diatoms, other phytoplankton groups such as silicoflagellates have an internal silica skeleton. Dictyocha is often a common component of the phytoplankton in the north Pacific Gyre (Venrick, 1992; Scharek et al., 1999), were nearly absent during this survey in the SE Pacific. The open SE Pacific is far from the terrestrial inputs of dissolved silicon. The Antarctic waters spread along the deep SE Pacific Ocean. The waters of the PCC showed a nitratesilicic acid ratio higher than 1 that may be associated to a Si-deficiency (Fig. 6). At the bottom of the euphotic zone the nitrate-silicic acid ratio was also higher than 1 (Fig. 6). Compared to the other oceanic regions, the SE Pacific seems to show deficiencies of the stock of silicic acid compared to other major nutrients. However, it is uncertain whether silicic acid is the main factor that determinate the dominant HNLC$\mathrm{PA}$ and the diatoms of the bottom of the euphotic zone in the SPG.

Leynaert et al. (2001) hypothesized that silicon and iron limitations may interact. Iron is an essential component for the synthesis of chlorophyll and the nitrate reductase of the primary producers. It is unknown whether the iron from the Marquesas Islands Archipelago is the main responsible of the increase of the abundance of Pseudo-nitzschia and/or the increase is mainly due to the entrainment of deep major nutrients due the "island mass effect" (Signorini et al., 1999). In the present study, offshore the Marquesas Islands Archipelago, the dominance of the small Pseudo-nitzschia decreased and larger centric diatoms such as $R$. bergonii showed higher abundance (Fig. 8). In the proximity of islands the major nutrients and iron inputs is expected to be more continuous, favouring smaller diatoms such as Pseudonitzschia. In offshore waters, the pulsating enrichment events may favour species with big vacuoles such as $R$. bergonii able to accumulate nutrients for oligotrophic periods. Several studies showed that the domoic acid produced by Pseudonitzschia binds iron and copper (Rue and Bruland, 2001; Wells et al., 2005). The domoid acid is a strong organic ligand that facilitates the iron uptake of Pseudo-nitzschia spp. According to Wells et al. (2005) this may explain why Pseudo-nitzschia spp. have persistent populations in oceanic HNLC regions.

Other characteristic of the HNLC diatoms is that they are restricted to surface waters and they did not form the typical sub-surface maximum as usual in other diatoms. For example species considered as deep flora such as Planktoniella sol (Sournia, 1982) showed a surface distribution in the HNLC regions of the present study. Under unfavourable surface conditions the diatoms are expected to sink to deep waters. However, in the transition of the warm HNLC region to oligotrophic conditions $\left(13^{\circ} 32^{\prime} \mathrm{S}-132^{\circ} 07^{\prime} \mathrm{W}\right)$ Rhizosolenia bergonii remained near the surface despite the nitrate stock was depleted (Figs. 7-8, Table 1). It is uncertain the factor that determinate the surface distribution of these diatoms. Near the surface, the irradiance is higher and atmospheric inputs of iron are available. Iron is required for the photosynthetic electron transport system and chlorophyll molecule biosynthesis. At deeper waters (low irradiance), it can be expected that diatoms will require the synthesis of more chlorophyll and electron transport system molecules. Several studies reported that the iron requirements for diatoms increase at sub-saturating irradiances and decrease the efficiency of the utilization of iron (Sunda and Huntsman, 1995, 1997; Muggli and Harrison, 1997). Consequently in irondepleted waters, the diatoms near the surface may grow with less iron requirements than in deeper waters. The hypothesis that the iron-stress would determinate the vertical distribution of HNLC diatoms requires further research. Other features such as the anti-grazing strategies and the physiology of HNLC species Pseudo-nitzschia delicatissima and Rhizosolenia bergonii need to be investigated.

Environmental factors, other than temperature and salinity, are responsible for the establishment and the persistence of a weakly silicified diatom assemblage in two geographically and hydrologically distant regions, the tropical central Pacific and the western limit of the Perú-Chile Current during the austral summer. The nitrate-silicic acid ratio ranging from 13 in the euphotic zone and frustules anomalously fragmented in Rhizosolenia bergonii suggest that Si-deficiency may be responsible of the HNLC-PA. Further studies are required to establish the factors that favour the occurrence of this distinctive phytoplankton assemblage.

Acknowledgements. D. Tailliez and C. Bournot are warmly thanked for their efficient help in CTD rosette management and data processing. We thank J. Ras for collection assistance. F. Gómez is supported by a post-doctoral grant of the Ministerio Español de Educación y Ciencia \#2007-0213. This is a contribution of the BIOSOPE project of the LEFE-CYBER program.

Edited by: S. Pantoja 


\section{References}

Avaria, S. and Muñoz, P.: Effects of the 1982-1983 El Niño on the marine phytoplankton off Northern Chile, J. Geophys. Res., 92, 14 369-14 382, 1987.

Balech, E.: Tintinnoidea y Dinoflagellata del Pacífico según material de las expediciones Norpac y Downwind del Instituto Scripps de Oceanografía, Rev. Mus. Argent. Cienc. Nat. "B. Rivadavia". Cienc. Zool., 7, 1-253, 1962.

Brzezinski, A. M.: The Si:C:N ratio of marine diatoms: interspecific variability and the effect of some environmental variables, J. Phycol., 21, 347-357, 1985.

Buck, K. R. and Chavez, F. P.: Diatom aggregates from the open ocean, J. Plankton Res., 16, 1149-1457, 1994.

Chavez, F. P.: Size distribution of phytoplankton in the central and eastern tropical Pacific, Global Biogeochem. Cycles, 3, 27-35, 1989.

Chavez, F. P. and Buck, K. R.: Phytoplankton taxa in relation to primary production in the equatorial Pacific, Deep-Sea Res., 37, 1733-1752, 1990.

Chavez, F. P., Buck, K. R., Coale, K. H., Martin, J. H., Ditullio, G. R., Welshchmeyer, N. A., Jacobsen, A. C., and Barber, R. T.: Growth rate, grazing, sinking, and iron limitation of equatorial Pacific phytoplankton, Limnol. Oceanogr., 36, 1816-1833, 1991.

Claustre, H. and Maritorena, S.: The many shades of ocean blue, Science, 302, 1514-1515, 2003.

Coale, K. H., Johnson, K. S., Fitzwater, S. E., et al.: A massive phytoplankton bloom induced by an ecosystem-scale iron fertilization experiment in the Equatorial Pacific Ocean, Nature, 383, 495-501, 1996.

DiTullio, G. R., Geesey, M. E., Jones, D. R., Daly, K. L., Campbell, L., and Smith, O. S.: Phytoplankton assemblage structure and primary productivity along $170^{\circ} \mathrm{W}$ in the South Pacific Ocean, Mar. Ecol. Prog. Ser., 255, 55-80, 2003.

Dugdale, R. C. and Wilkerson, F. P.: Silicate regulation of new production in the equatorial Pacific upwelling, Nature, 391, 270273, 1998.

Frost, B. W. and Franzen, N. C.: Grazing and iron limitation in the control of phytoplankton stock and nutrient concentration: a chemostat analogue of the Pacific equatorial upwelling zone, Mar. Ecol. Progr. Ser., 83, 291-303, 1992.

Fryxell, G. A. and Kaczmarska, I.: Specific variability in Fe enrichment cultures from the equatorial Pacific, J. Plankton Res., 16, 755-769, 1995.

Hardy, J., Hanneman, A., Behrenfeld, M., and Horner, R.: Environmental biogeography of near-surface phytoplankton in the southeast Pacific Ocean, Deep-Sea Res. I, 43, 1647-1659, 1996.

Hasle, G. R.: Phytoplankton and ciliate species from the tropical Pacific, Skrifter utgitt av Det Norske Videnskaps-Akademi i Oslo. I, Matematisk-Naturvideskabelig Klasse, 2, 1-50, 1960.

Ianora, A., Poulet, S. A., and Miralto, A.: The effects of diatoms on copepod reproduction: A review, Phycologia, 42, 351-363, 2003.

Iriarte, J. L. and Fryxell, G. A.: Microplankton at the equatorial Pacific $\left(140^{\circ} \mathrm{W}\right)$ during the JGOFS EqPac Time Series studies: March to April and October 1992, Deep-Sea Res. II, 42, 559583, 1995.

Kaczmarska, I. and Fryxell, G. A.: The genus Nitzschia: Three new species from the equatorial Pacific Ocean, Diatom. Res., 9, 8798, 1994.
Kolbe, R. W.: Diatom from the Equatorial Pacific cores, Swedish Deep-Sea Exped. 1947-1948, Repts. 6, 3-49, 1954.

Landry, M. R., Constantinou, J., and Kirshtein, J.: Microzooplankton grazing in the central equatorial Pacific during February and August, 1992, Deep-Sea Res. II, 42, 657-671, 1995.

Leising, A. W., Gentleman, W. C., and Frost, B. W.: The threshold feeding response of microzooplankton within Pacific highnitrate low-chlorophyll ecosystem models under steady and variable iron input, Deep-Sea Res. II, 50, 2877-2894, 2003.

Leynaert, A., Tréguer, P., Christiane Lancelot, C., and Rodier, M.: Silicon limitation of biogenic silica production in the Equatorial Pacific, Deep-Sea Res., I 48, 639-660, 2001.

Longhurst, A. R.: Ecological Geography of the Sea, Academic Press, London, 1998.

Muggli, D. L. and Harrison, P. J.: Effects of iron on two oceanic phytoplankters grown in natural NE subarctic Pacific seawater with no artificial chelators present, J. Exp. Mar. Biol. Ecol., 212, 225-237, 1997.

Raimbault, P., Garcia, N. and Cerutti, F.: Distribution of inorganic and organic nutrients in the South Pacific Ocean - evidence for long-term accumulation of organic matter in nitrogen depleted waters. Biogeosciences Discuss., 4, 3041-3087, 2007

Rivera, P. A.: Guide for references and distribution for the class Bacillariophyceae in Chile between $18^{\circ} 28^{\prime} \mathrm{S}$ and $58^{\circ} \mathrm{S}$, Bibliotheca Diatomologica, 3, 1-386, 1983.

Rodríguez, L., Escribano, R., Grone, G., Irribarren, C., and Castro, H.: Ecología del fitoplancton en la Bahía de Antofagasta (23 ${ }^{\circ}$ S), Chile, Rev. Biol. Mar. Valparaíso, 31, 65-80, 1996.

Roman, M. R. and Gauzens, A. L.: Copepod grazing in the equatorial Pacific, Limnol. Oceanogr., 42, 623-634, 1997.

Rue, E. L. and Bruland, K. W.: Domoic acid binds iron and copper: a possible role for the toxin produced by the marine diatom Pseudo-nitzschia, Mar. Chem., 76, 127-134, 2001.

Scharek, R., Latasa, M., Karl, D. M., and Bidigare, R. R.: Temporal variations in diatom abundance and downward vertical flux in the oligotrophic North Pacific gyre, Deep-Sea Res., I 46, 1051-1075, 1999.

Schneider, W., Fuenzalida, R., Rodríguez-Rubio, E., GarcésVargas, J., and Bravo, L.: Characteristics and formation of Eastern South Pacific Intermediate Water, Geophys. Res. Lett., 20 30, 1581, doi:10.1029/2003GL017086, 2003.

Signorini, S. R., McClain, C. R., and Dandonneau, Y.: Mixing and phytoplankton bloom in the wake of the Marquesas Islands, Geophys. Res. Lett., 26, 3121-3124, 1999.

Sournia, A.: Is there a shade flora in the marine plankton?, J. Plankton Res., 4, 391-399, 1982.

Sunda, W. G. and Huntsman, S. A.: Iron uptake and growth limitation in oceanic and coastal phytoplankton, Mar. Chem., 50, 189-206, 1995.

Sunda, W. G. and Huntsman, S. A.: Interrelated influence of iron, light and cell size on marine phytoplankton growth, Nature, 390, 389-392, 1997.

Venrick, E. L.: Phytoplankton in an oligotrophic ocean: observations and questions, Ecol. Monogr., 52, 129-154, 1982.

Wells, M. L., Trick, C. G., Cochlan, W. P., Hughes, M. P. and Trainer, V. L.: Domoic acid: The synergy of iron, copper, and the toxicity of diatoms, Limnol. Oceanogr., 50, 1908-1917, 2005. 